The Journal of Laryngology \& Otology

http://journals.cambridge.org/JLO

Additional services for The Journal of Laryngology \& Otology:

Email alerts: $\underline{\text { Click here }}$

Subscriptions: $\underline{\text { Click here }}$

Commercial reprints: $\underline{\text { Click here }}$

Terms of use : $\underline{\text { Click here }}$

\title{
Wegener's granulomatosis of the orbit: two cases requiring endoscopic surgical decompression
}

J M Fishman, A Slovick and C A East

The Journal of Laryngology \& Otology / Volume 122 / Issue 11 / November 2008, pp 1257 - 1259

DOI: 10.1017/S0022215108001953, Published online: 09 April 2008

Link to this article: http://journals.cambridge.org/abstract_S0022215108001953

How to cite this article:

J M Fishman, A Slovick and C A East (2008). Wegener's granulomatosis of the orbit: two cases requiring endoscopic surgical decompression. The Journal of Laryngology \& Otology, 122, pp 1257-1259 doi:10.1017/S0022215108001953

Request Permissions : $\underline{\text { Click here }}$ 


\title{
Wegener's granulomatosis of the orbit: two cases requiring endoscopic surgical decompression
}

\author{
J M Fishman, A Slovick, C A East*
}

\begin{abstract}
Objectives: We report two unusual and contrasting cases of Wegener's granulomatosis involving the orbit, requiring urgent endoscopic orbital decompression surgery.

Case series: Both patients presented with an acute deterioration in visual function which failed to improve with medical management. Case one presented with systemic features of the condition, in contrast with case two, who presented with a more localised form of the disease. Whereas case one exhibited primary orbital disease with maxillary sinus extension (i.e. focal disease), case two illustrates orbital involvement secondary to sinus disease extension (i.e. contiguous disease). Prompt diagnosis, assisted by the presence of systemic features, led to a good visual outcome in case one. In case two, in which diagnosis was difficult and surgery delayed, the outcome was poor.

Conclusions: These cases of orbital Wegener's granulomatosis illustrate the diagnostic challenge, the requirement for early intervention following acute visual deterioration and the importance of heightened awareness of the rarer ENT manifestations of this disease.
\end{abstract}

Key words: Wegener's Granulomatosis; Orbit; Paranasal Sinuses; Visual Loss

\section{Introduction}

Wegener's granulomatosis is a rare, multisystemic, granulomatous inflammatory condition of unknown aetiology which classically involves the upper and lower respiratory tracts and kidneys. Ninety per cent of patients with Wegener's granulomatosis have head and neck involvement, mostly of the sinuses, so otolaryngologists play a central role in the diagnosis and management of the condition.

Ocular involvement is a well described entity affecting 50-60 per cent of Wegener's granulomatosis patients throughout their disease course, either as part of systemic disease or a more localised form. Orbital disease may result either from primary disease or from sinus disease extension. In addition, 8-16 per cent of patients have ocular disease at the time of diagnosis, if it is not the presenting feature. ${ }^{1}$

Wegener's granulomatosis is notoriously difficult to diagnose clinically, serologically, histopathologically and radiologically. Patients who present with head and neck manifestations of the disease have been shown to have a considerably delayed diagnosis (nine versus 5.9 months). ${ }^{1}$ In particular, the greatest delay in Wegener's granulomatosis diagnosis occurs when the presenting complaint is orbital rather than non-orbital in nature $(15$ months versus nine months for sinonasal disease).

Despite frequent ENT involvement and improved diagnostic testing, awareness of Wegener's granulomatosis amongst UK ENT surgeons has not increased over the last three decades. ${ }^{1}$ Delayed diagnosis and management can lead to serious visual morbidity; shown to occur in 50 per cent of patients with Wegener's granulomatosis. ${ }^{2}$
Here, we present two cases of orbital Wegener's granulomatosis which required orbital decompression as a sightsaving, emergency procedure.

\section{Case one}

A 35-year-old man with systemic Wegener's granulomatosis presented to the emergency department with acute left eye pain and blurred vision. A similar episode, one month earlier, had been controlled with prednisolone, mycophenolate mofetil and cyclophosphamide.

Examination of the left eye revealed scleritis and mild proptosis. There was reduced upward gaze (30 per cent), visual acuity (to counting fingers) and colour vision (Ishihara left $0 / 17$, right $17 / 17$ ), with a sluggish pupil response and a relevant afferent pupillary defect. Fundal vessel tortuosity and choroidal folds were seen. No abnormalities of the right eye were identified.

Gadolinium-enhanced magnetic resonance imaging (MRI) of the orbits showed intra- and extra-conal abnormal tissue encasing the optic nerve, with prolapse into the left maxillary sinus, consistent with orbital Wegener's granulomatosis (Figure 1).

The patient was commenced on intravenous dexamethasone but failed to improve. He underwent emergency endoscopic left orbital decompression and biopsy the next day.

Post-operative improvements were seen in visual acuity (6/24 versus finger counting), colour vision and pupillary response. Histological analysis was consistent with Wegener's granulomatosis.

From the Department of Otolaryngology, The Royal Free Hospital, and the *Royal National Throat, Nose and Ear Hospital, London, UK.

Accepted for publication: 20 December 2007. First published online 9 April 2008. 
(a)

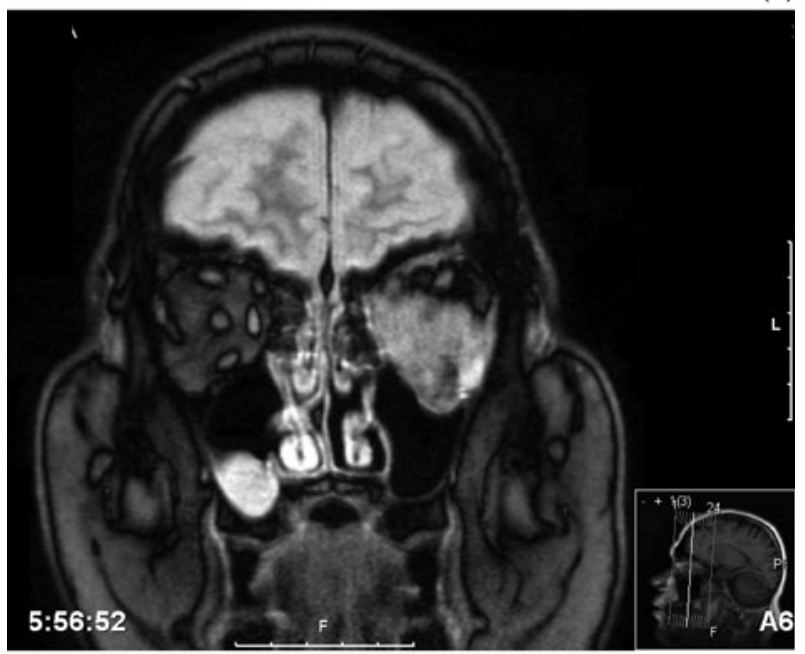

(b)

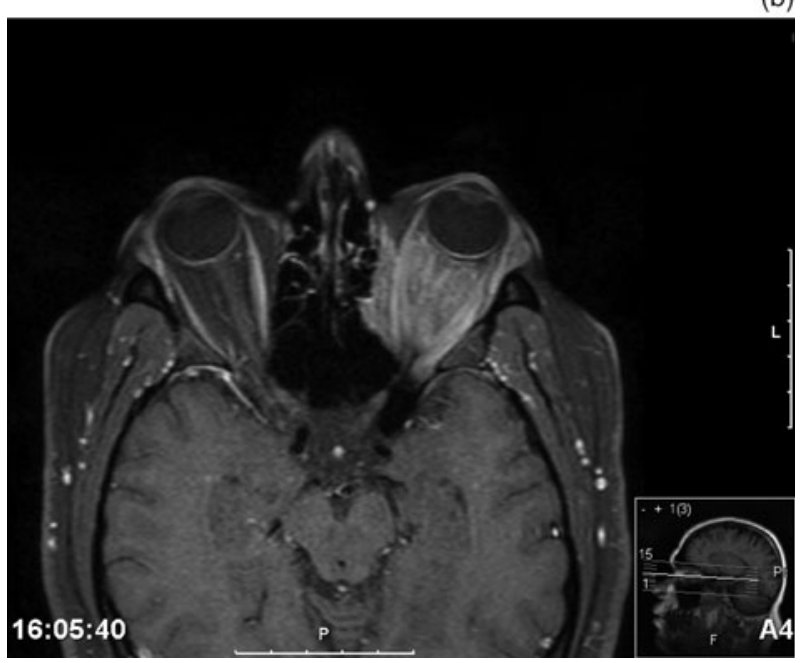

FIG. 1

Gadolinium-enhanced magnetic resonance imaging scans of the orbits and head; (a) coronal and (b) axial views. There is expansion of the left orbit, associated with some medial displacement of the lamina papyracea and some depression of the floor. There is extensive abnormal tissue within the left orbit, both within the muscle cone and also extraconal. There is prolapse of this tissue into the upper part of the left maxillary sinus. A little nodular protuberance of the tissue is noted at the left lateral canthus. Maximal compromise of the optic nerve is presumably at the apex. The right optic nerve sleeve complex is unusually straight, and there is rather abundant right orbital fat, possibly producing a little proptosis. The appearance is of extensive left orbital abnormality with some changes in the medial and inferior orbital walls. The radiological appearances cannot be considered specific, but are compatible with a granulomatous disease process.

\section{Case two}

A 66-year-old man with no known history of Wegener's granulomatosis presented acutely with right eye pain and blurred vision.

Two years earlier, the patient had presented to the ENT department with recurrent right-sided epistaxis, sinusitis and a friable, right-sided mass. A computed tomography (CT) scan of the sinuses at the time had shown right-sided opacifications of the frontal, maxillary and ethmoidal sinuses. Biopsies of the right middle meatus and lateral nasal wall had been non-diagnostic on two occasions. Analysis for cytoplasmic antineutrophilic cytoplasmic antibodies (cANCA) had been positive. No diagnosis had been reached, and the patient had been lost to follow up.

On examination during the current presentation, there was reduced right-sided visual acuity (6/18 right, 6/9 left with pinhole) and movement (50 per cent abduction, 0 per cent adduction, 30 per cent up- and downward gaze). Colour vision, pupil reactions and fundi were normal.

Analyses for serum angiotensin-converting enzyme and cANCA were negative.

A chest CT showed pulmonary nodules suggestive of Wegener's granulomatosis. An MRI revealed soft tissue in the right maxillary sinus with some in the orbit. This was suspicious of Wegener's granulomatosis in view of the earlier biopsy findings.

Another biopsy was taken, showing non-specific granulomata, but no definitive diagnosis was made.

The patient improved on high dose prednisolone and cyclophosphamide treatment and was discharged.

One month later, the patient was readmitted with right eye pain and reduced visual acuity (to hand movements). The right-sided, direct pupillary reflex was sluggish and eye movement considerably reduced. An MRI showed a mass in the right ethmoid sinus, extending intra-orbitally (Figure 2).

Endoscopic right anterior orbital decompression was performed on this occasion. Pain and visual acuity initially improved, but there was sudden post-operative pain and

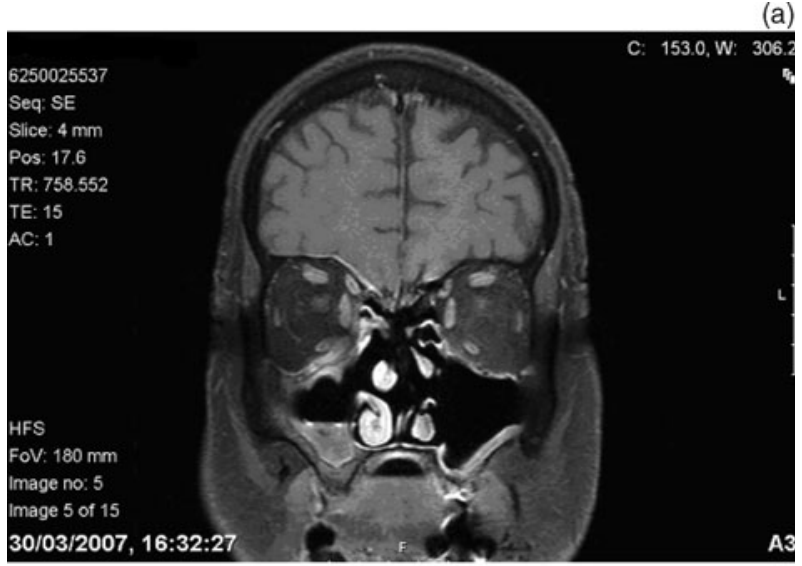

(b)

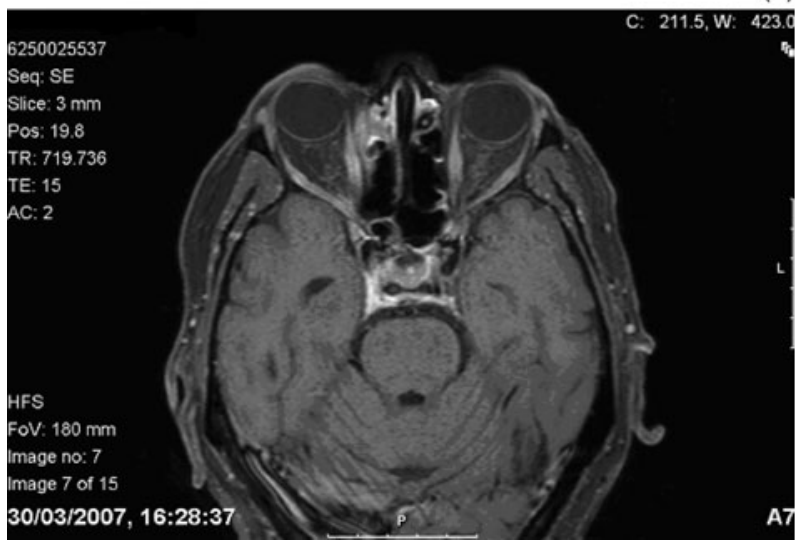

FIG. 2

Gadolinium-enhanced magnetic resonance imaging scans of the head; (a) coronal and (b) axial views. The scans show a mass lying between the abnormal mucosa in the right anterior ethmoid cells and the medial rectus muscle. The lesion is intra-orbital and intraconal, and is presumably an extension of the inflammatory process within the paranasal sinuses. The lacrimal glands are not enlarged, making Wegener's granulomatosis more likely than sarcoidosis. The appearance of the left orbital contents is within normal limits. 
visual deterioration (to $3 / 60$ ) necessitating granuloma excision, thereby relieving the optic nerve compression. Postoperative visual acuity on the right was $6 / 36$, with a sluggish pupil and reduced upward gaze. The patient was commenced on rituximab, since the condition seemed resistant to other forms of medical therapy.

External histological consultation was sought, and the clinical picture was felt to fit best with Wegener's granulomatosis disease.

\section{Discussion}

Wegener's granulomatosis is a severe, non-infectious, granulomatous and necrotising vasculitis with numerous otorhinolaryngological manifestations. Both the cases described above demonstrated orbital complications of Wegener's granulomatosis, case one as part of a multisystemic disease and case two as a more localised form without renal involvement. The aetiology of orbital disease is two-fold, as illustrated by our two cases. Case one had primary orbital disease with maxillary sinus extension (i.e. focal disease), while case two had orbital involvement secondary to sinus disease extension (i.e. contiguous disease). The presenting symptoms of unilateral blurred vision and eye pain were unusual; bilateral eye disease is frequently reported. ${ }^{2}$

Wegener's granulomatosis of the head and neck is difficult to diagnose clinically. This is especially true of the orbit, due to the disease's rarity at this site and non-specific presentation. Combined consideration of clinical, serological, radiological and histopathological findings is vital in achieving a successful diagnosis. For example, the cytoplasmic antineutrophilic cytoplasmic antibody (cANCA) test is reported to offer 91 per cent sensitivity and 99 per cent specificity for multisystemic Wegener's granulomatosis. ${ }^{3}$ However, this drops to 50-60 per cent sensitivity in localised disease. ${ }^{3}$ This is exemplified by case two, in which cANCA had been positive two years prior to presentation, but was later negative. An MRI scan of the orbits and CT scan of the sinuses helped to define the extent of the disease, but demonstrated only non-specific inflammatory changes.

- Ocular involvement in Wegener's granulomatosis is a well described entity, affecting 50-60 per cent of patients throughout their disease course

- This paper describes two unusual and contrasting cases of Wegener's granulomatosis involving the orbit, requiring urgent endoscopic orbital decompression surgery

- Both patients presented with an acute deterioration in visual function, which failed to improve with medical management

- The authors emphasise the requirement for early intervention following acute visual deterioration, and the importance of heightened ENT awareness of this rarer presentation of Wegener's granulomatosis

Biopsies may be non-diagnostic in up to 50 per cent of specimens. ${ }^{4}$ Case two required two repeat biopsies. The classical triad seen in Wegener's granulomatosis, of parenchymal necrosis, vasculitis and granulomatous inflammation, varies according to tissue type, and has been identified in 91 per cent of lung biopsies versus 54 per cent of orbital biopsies. ${ }^{4}$ The diverse pathological features described in cases one and two above demonstrate the challenge of achieving an accurate histopathological diagnosis of orbital Wegener's granulomatosis.
Early diagnosis is critical, preventing delayed intervention and irreversible morbidity. Vision was preserved in case one, as the diagnosis was known, facilitating a prompt decision regarding orbital decompression surgery. In cases in which diagnosis is difficult and surgery delayed, as in case two, outcome is often poor.

Case two had presented to the ENT department two years earlier with epistaxis, positive cANCA serology and MRI changes, yet no diagnosis had been made. This correlates with the findings of Srouji et al., who showed that 56 per cent of Wegener's granulomatosis patients presenting to the ENT department with head and neck symptoms were not diagnosed at presentation. ${ }^{1}$ The average diagnostic delay was greater in those patients with head and neck symptoms than in those without head and neck manifestations of the disease (nine months versus 5.9 months). ${ }^{1}$

Pakrou et al. have suggested that periodic ophthalmic assessment is of value in Wegener's granulomatosis. Patients with systemic Wegener's granulomatosis are at risk of eye involvement, whilst those with initially localised ocular Wegener's granulomatosis can develop systemic disease. Case two had initially been lost to follow up. Regular review may have detected earlier visual deterioration, avoiding the need for emergency surgery.

\section{Conclusions}

Here, we demonstrate the continued diagnostic challenge posed to otolaryngologists by Wegener's granulomatosis. Orbital involvement with acute visual deterioration requires immediate orbital decompression in combination with systemic immunosuppressive treatment, in order to preserve visual function.

Diagnostic and treatment advances are still ongoing. Even so, otolaryngologists should be made more aware of the orbital complications of Wegener's granulomatosis and its potential for orbital spread from the sinuses. A close collaboration between ophthalmologists and otolaryngologists is mandatory. Regular review may improve detection of early visual changes, thereby reducing the current visual morbidity rate of 50 per cent.

\section{References}

1 Srouji IA, Andrews P, Edwards C, Lund VJ. Patterns of presentation and diagnosis of patients with Wegener's granulomatosis: ENT aspects. J Laryngol Otol 2007;121:653-8

2 Pakrou N, Selva D, Leibovitch I. Wegener's granulomatosis: ophthalmic manifestations and management. Semin Arthritis Rheum 2006:35:284-92

3 Rao JK, Weinberger M, Oddone EZ, Allen NB, Landsman $\mathrm{P}$, Feussner JR. The role of antineutrophil cytoplasmic antibody (c-ANCA) testing in the diagnosis of Wegener granulomatosis. Ann Intern Med 1995;123:925-32

4 Kalina PH, Lie JT, Campbell RJ, Garrity JA. Diagnostic value and limitations of orbital biopsy in Wegener's granulomatosis. Ophthalmology 1992;99:120-4

Address for correspondence:

Mr Jonathan Fishman,

Department of Otolaryngology,

The Royal Free Hospital,

Pond Street,

London NW3 2QG, UK.

Fax: 02078302422

E-mail: jfishman@doctors.org.uk

Mr J Fishman takes responsibility for the integrity of

the content of the paper.

Competing interests: None declared 